Original Research Paper

\title{
Adapted Newton-Kantorovich Methods for Nonlinear Integral Equations
}

\author{
Mostefa Nadir and Amina Khirani \\ Department of Mathematics, University of Msila, 28000, Algeria
}

\author{
Article history \\ Received: 18-11-2015 \\ Revised: 05-12-2015 \\ Accepted: 02-07-2016 \\ Corresponding Author: \\ Mostefa Nadir \\ Department of Mathematics, \\ University of Msila, 28000, \\ Algeria \\ Email: mostefanadir@yahoo.fr
}

\section{Introduction}

Nonlinear integral equation is an important branch in contemporary mathematics and arises many applied areas which include engineering problems, such as mechanics, physics, astronomy, biology, economics, potential theory and electrostatics (Golberg, 1990; Nadir and Gagui, 2014). Those equations are classified into Fredholm and Volterra equations following the upper bound of the region for the integral part is constant or variable. Many different methods are used to obtain the numerical solution of the nonlinear integral equations (Nadir and Rahmoune, 2007; Polyanin and Manzhirov, 2008):

$$
\varphi(s)-\int_{\Omega} k(s, t, \varphi(t)) d t=f(s), s, t \in \Omega
$$

where, the functions $f(s)$ and $k(s, t, \varphi)$ are given and continuous functions in $\Omega$ and $D(k)=\Omega \times \Omega \times I,(I \in R)$, respectively, the function $\varphi(t)$ is to be determined as continuous function $\Omega \rightarrow I$.

For the solution of the nonlinear integral equations we present specifics conditions for the existence of this ones:

- $k(s, t, \varphi(t)): \Omega \times \Omega \times I \rightarrow R$ is continuous and bounded in this domain

- $k(s, t, \varphi)$ : Is Lipschitzian for the third variable. In other words there exists $L>0$ such that:

$$
\left|k\left(s, t, \varphi_{1}\right)-k\left(s, t, \varphi_{2}\right)\right| \leq L\left|\varphi_{1}-\varphi_{2}\right| \quad s, t \in \Omega \varphi_{1}, \varphi_{2} \in I
$$

- $f: \Omega \rightarrow R$ is continuous

- $\frac{1}{L(b-a)}>1$

Depending on $\Omega=[a, s]$ or $\Omega=[a, b]$ the Equation 1 is a nonlinear Volterra or Fredholm integral equation, respectively.

We apply the Newton-Kantorovich method to the general nonlinear integral equation:

$$
P(\varphi)=\varphi(s)-\int_{\Omega} k(s, t, \varphi(t)) d t-f(s)
$$

It is known that, the fact where the function $k(s, t, \varphi)$ is continuous and Lipshitzian for the third variable then, $P(\varphi)$ is Fréchet differentiable mapping of a Banach spaces $C(\Omega)$ into itself for all $f(s) ; \varphi(s) \in C(\Omega)$, say:

$$
\begin{aligned}
& G D(P ; \varphi) h(s)=P^{\prime}(\varphi) h(s)=h(s) \\
& -\int_{\Omega} k_{\varphi}(s, t, \varphi(t)) h(t) d t
\end{aligned}
$$

where, $k_{\varphi}(s, t, \quad \varphi(t))$ designates the derivative $\frac{\partial k}{\partial \varphi}(s, t, \varphi(t))$.

For the resolution of the functional equation $P(\varphi)=0$ where $P$ is Fréchet differentiable on a convex set of a Banach space $C(\Omega)$, Kantorovich imitates the Newton method for the equation of the tangent line given by the first two terms of Taylor's formula, written as the method of successive approximation: 


$$
P\left(\varphi_{n+1}\right)=P\left(\varphi_{n}\right)+P^{\prime}\left(\varphi_{n}\right)\left(\varphi_{n+1}-\varphi_{n}\right)=0
$$

Or equivalently:

$$
P^{\prime}\left(\varphi_{n}\right)\left(\varphi_{n+1}-\varphi_{n}\right)=-P\left(\varphi_{n}\right)
$$

The explicit form to the Equation 3 is given as:

$$
\begin{aligned}
& \varphi_{n}(s)-\int_{\Omega} k\left(s, t, \varphi_{n}(t)\right) d t-f(s)+\left(\varphi_{n+1}(s)-\varphi_{n}(s)\right) \\
& -\int_{\Omega} k_{\varphi}\left(s, t, \varphi_{n}(t)\right)\left(\varphi_{n+1}(t)-\varphi_{n}(t)\right) d t=0
\end{aligned}
$$

Or still:

$$
\begin{aligned}
& \varphi_{n+1}(s)=f(s)-\int_{\Omega} k\left(s, t, \varphi_{n}(t)\right) d t \\
& +\int_{\Omega} k_{\varphi}\left(s, t, \varphi_{n}(t)\right)\left(\varphi_{n+1}(t)-\varphi_{n}(t)\right) d t
\end{aligned}
$$

In the Newton-Kantorovich method, we remark that, the kernels $k\left(s, t, \varphi_{n}(t)\right)$ and $k_{\varphi}\left(s, t, \varphi_{n}(t)\right)$ of the righthand side of the Equation 4 are replaced by the ones $k(s$, $\left.t, \varphi_{0}(t)\right)$ and $k_{\varphi}\left(s, t, \varphi_{0}(t)\right)$ where $\varphi_{0}$ represents the initial value so that, the Equation 4 becomes a linear integral equation. However, in our work we treat the Equation 4 by adapted a modification, where we replace the expression $\left(\varphi_{n+1}(t)-\varphi_{n}(t)\right)$ in the right-hand side by the one $\left(\varphi_{n}(t)-\varphi_{n-1}(t)\right)$ so that, the Equation 4 becomes:

$$
\begin{aligned}
& \varphi_{n+1}(s)=f(s)-\int_{\Omega} k\left(s, t, \varphi_{n}(t)\right) d t \\
& +\int_{\Omega} k_{\varphi}\left(s, t, \varphi_{n}(t)\right)\left(\varphi_{n}(t)-\varphi_{n-1}(t)\right) d t
\end{aligned}
$$

The first approximation $\varphi_{1}(s)$ is obtained by substituting the initial approximation $\varphi_{0}(s)=f(s)$ into the right hand side of the integral equation, giving:

$$
\begin{aligned}
& \varphi_{n}(s)=f(s)-\int_{\Omega} k(s, t, f(s)) d t \\
& +\int_{\Omega} k_{\varphi}(s, t, f(s))(f(s)-0) d t
\end{aligned}
$$

And so on, higher iterates may be defined by Equation 4 where we approximate the two integrals presented in Equation 4 by one of the basic numerical integration formulas such as trapezoid method, Simpson methods, or Gauss methods.

\section{Convergence and Applications Theorem}

Let $P$ be an operator defined on a Banach space $\mathrm{E}$ into a Banach space $F$ and Fréchet differentiable for $\varphi \in \Omega$ an open convex set in $\mathrm{E}$, satisfies the following conditions:

$$
\begin{aligned}
& \text { (A1) }\left\|P^{\prime}(\varphi)-P^{\prime}(\psi)\right\| \leq L\|\varphi-\psi\|, \varphi, \psi \in \Omega \\
& (A 2)\left\|\left[P^{\prime}\left(\varphi_{0}\right)\right]^{-1}\right\| \leq M, \varphi_{0} \in \Omega \\
& (A 3)\left\|\left[P^{\prime}\left(\varphi_{0}\right)\right]^{-1} P\left(\varphi_{0}\right)\right\| \leq N, \varphi_{0} \in \Omega
\end{aligned}
$$

With the constants $L, M$ and $N$ satisfying $L M<1$, $L M N \leq \frac{1}{2}$ then there exists a domain $\Omega_{1}=\left\{\varphi ;\left\|\varphi-\varphi_{0}\right\| \leq h=\frac{(\sqrt{2}-1)}{L M \sqrt{2}}\right\} \subset \Omega \quad$ such that, the successive approximations:

$$
\varphi_{n+1}=\varphi_{n}-\left[P^{\prime}\left(\varphi_{n}\right)\right]^{-1} P\left(\varphi_{n}\right)
$$

Are defined for all $n, \varphi_{n} \in \Omega_{1}, n=1,2, \ldots$ and converge to the exact solution $\varphi \in \Omega_{1}$ which satisfies $P(\varphi)=0$ : Further:

$$
\left\|\varphi_{n}-\varphi\right\| \leq \frac{C}{2^{n}(L M N)}, C \in \mathbb{R}_{+}^{*}, n=1,2,3 \ldots \ldots
$$

\section{Proof}

Indeed, it is easy to see that:

$$
\begin{aligned}
& P(\varphi)-P(\psi)=\int_{0}^{1} P^{\prime}(\varphi+t(\varphi-\psi))(\varphi-\psi) d t \\
& =\int_{0}^{1}\left(P^{\prime}(\varphi+t(\varphi-\psi))-P^{\prime}(\varphi)\right)(\varphi-\psi) d t \\
& +\int_{0}^{1} P^{\prime}(\varphi)(\varphi-\psi) d t \\
& \left\|P(\varphi)-P(\psi)-P^{\prime}(\varphi)(\varphi-\psi)\right\| \\
& =\left\|\int_{0}^{1}\left(P^{\prime}(\varphi+t(\varphi-\psi))-P^{\prime}(\varphi)\right)(\varphi-\psi) d t\right\| \\
& \left\|P(\varphi)-P(\psi)-P^{\prime}(\varphi)(\varphi-\psi)\right\| \\
& \leq \int_{0}^{1}\left\|P^{\prime}\left(\varphi+t(\varphi-\psi)-P^{\prime}(\varphi)\right)\right\|(\varphi-\psi) \| d t
\end{aligned}
$$

Using condition (A1), on $\Omega$ we obtain:

$$
\left\|P(\varphi)-P(\psi)-P^{\prime}(\varphi(\varphi-\psi))\right\| \leq \frac{L}{2}\|(\varphi-\psi)\|^{2}
$$

Also, for $\varphi \in \Omega_{1}$, we get:

$$
\left\|P^{\prime}(\varphi)-P^{\prime}\left(\varphi_{0}\right)\right\| \leq L\left\|\varphi-\varphi_{0}\right\| \leq L h<\frac{1}{M}
$$

Then, the relation (7) shows that $P^{\prime}(\varphi)$ is invertible for all $\varphi \in \Omega_{1}$ and it comes: 


$$
\left[P^{\prime}(\varphi)\right]^{-1}-\left(I-\left[P^{\prime}\left(\varphi_{0}\right)\right]^{-1}\left(P^{\prime}(\varphi)-P^{\prime}\left(\varphi_{0}\right)\right)^{-1}\left[P^{\prime}\left(\varphi_{0}\right)\right]^{-1}\right.
$$

Or still:

$$
\left\|\left[P^{\prime}(\varphi)\right]^{-1}\right\| \leq \frac{M}{(1-L M)\left\|\varphi-\varphi_{0}\right\|}
$$

Given the Newton function as:

$$
N(\varphi)=\varphi-\left[P^{\prime}(\varphi)\right]^{-1} P(\varphi)
$$

With $\varphi$ and $N(\varphi)$ in $\Omega_{1}$ and $\varphi_{n+1}=N\left(\varphi_{n}\right)$, we get:

$$
\|N(N(\varphi))-N(\varphi)\|=\left[P^{\prime}(N(\varphi))\right]^{-1} P(N(\varphi))
$$

Hence:

$$
\|P(N(\varphi))\| \leq \frac{L}{2}\|N(\varphi)-\varphi\|^{2}
$$

On the other hand

$$
\left\|P^{\prime}(N(\varphi))\right\|^{-1} \leq \frac{M}{(1-L M)\left\|N(\varphi)-\varphi_{0}\right\|}
$$

From the relations (9) and (10) we obtain:

$$
\|N(N(\varphi))-N(\varphi)\| \leq \frac{L M\|N(\varphi)-\varphi\|^{2}}{2(1-L M)\left\|N(\varphi)-\varphi_{0}\right\|}
$$

or still:

$$
\begin{aligned}
& \left\|\varphi_{n+1}-\varphi_{n}\right\| \leq \frac{L M\left\|\varphi_{n}-\varphi_{n-1}\right\|^{2}}{2(1-L M)\left\|\varphi_{n}-\varphi_{0}\right\|} \\
& \leq \frac{(L M)^{n}\left\|\varphi_{1}-\varphi_{0}\right\|^{2 n}}{2^{n}(1-L M)^{n}\left\|\varphi_{1}-\varphi_{0}\right\|^{n}}
\end{aligned}
$$

From the relation $\left\|\varphi_{q}-\varphi_{p}\right\| \leq\left\|\varphi_{q}-\varphi_{q-1}\right\|+\| \varphi_{q-1}-\varphi_{q-}$ ${ }_{2}\|+\ldots+\| \varphi_{p+1}-\varphi_{p} \|$ it comes the sequence $\varphi_{n}$ is Cauchy sequence in Banach space. Thus this sequence $\varphi_{n}$ represents the Newton iterations are defined and converges to the solution $\varphi$ in $\Omega_{1}$ (Wouk, 1979):

\section{Illustrating Examples}

\section{Example 1}

Consider the nonlinear integral equation of Volterra:

$$
\varphi(s)-\int_{0}^{s} \sin \varphi(t) d t=s+\cos s-1,0 \leq s, t \leq 1
$$

where, the function $f\left(t_{0}\right)$ is chosen so that the exact solution is given by:

$$
\varphi(t)=t
$$

The approximate solution $\tilde{\varphi}(t)$ of $\varphi(t)$ is obtained by the adapted Newton-Kantorovich method.

\section{Example 2}

Consider the nonlinear integral equation of Volterra:

$$
\varphi(s)-\int_{0}^{s} \varphi^{2}(t) d t=\exp (s)-\frac{1}{2}(\exp (2 s)-1), 0 \leq s, t \leq 1
$$

where, the function $f\left(t_{0}\right)$ is chosen so that the exact solution is given by:

$$
\varphi(t)=\exp (t)
$$

The approximate solution $\tilde{\varphi}(t)$ of $\varphi(t)$ is obtained by the adapted Newton-Kantorovich method.

\section{Example 3}

Consider the nonlinear integral equation of Volterra:

$$
\varphi(s)-\int_{0}^{s} \frac{1}{2} \varphi^{2}(t) d t=\sin s+\frac{1}{8} \sin 2 s-\frac{1}{4} s, 0 \leq s, t \leq 1
$$

where, the function $f\left(t_{0}\right)$ is chosen so that the exact solution is given by:

$$
\varphi(t)=\sin t
$$

The approximate solution $\tilde{\varphi}(t)$ of $\varphi(t)$ is obtained by the adapted Newton-Kantorovich method.

\section{Example 4}

Consider the nonlinear integral equation of Fredholm:

$$
\varphi(t)-\int_{0}^{1} \frac{t^{2} x^{2}}{1+\varphi^{2}(x)} d x-\left(\frac{1}{2}-\ln (2)\right) t^{2}+\sqrt{t}, 0 \leq x, t \leq 1
$$

where, the function $f\left(t_{0}\right)$ is chosen so that the exact solution is given by:

$$
\varphi(t)=\sqrt{t}
$$


The approximate solution $\tilde{\varphi}(t)$ of $\varphi(t)$ is obtained by the adapted Newton-Kantorovich method.

\section{Example 5}

Consider the nonlinear integral equation of Fredholm:

$$
\varphi(t)-\frac{1}{5} \int_{0}^{1} \cos (\pi t) \sin (\pi x) \varphi^{3}(x) d x=\sin (\pi t), 0 \leq x, t \leq 1
$$

where, the function $f\left(t_{0}\right)$ is chosen so that the exact solution is given by:

$$
\varphi(t)=\sin \pi t+\frac{1}{3}(20-\sqrt{391}) \cos \pi t
$$

The approximate solution $\mathrm{e}^{\prime}(\mathrm{t})$ of ' $(\mathrm{t})$ is obtained by the adapted Newton-Kantorovich method.

\section{Conclusion}

A numerical method for solving nonlinear Volterra and Fredholm integral equations, based on an adapted
Newton-Kontorovich methods is presented. The efficiency of this method is tested by solving some examples for which the exact solution is known. This allows us to estimate the exactness with our numerical results and compare those with another results. For nonlinear volterra integral equations our method is compared with the ones, the Haar wavelets and collocation, the fixed point technique with cubic Bspline scaling function, Adomian decomposition method and block pulse functions by collocation method. treated by (Babolian and Shahsavaran, 2007) Table 1, (Maleknejad et al., 2013) Table 2, (Awawdeh et al., 2009) Table 3 and (Shahsavaran, 2011) Table 4 respectively. On the other hand for nonlinear Fredholm integral equations our method is compared with the ones, the Haar wavelet method, the Urysohn form by Newton-Kantorovich-quadrature method and A numerical method treated by ((Lepik and Tamme, 2007)) Table 5, (Saberi-Nadja and Heidari, 2010) Table 6 and (Awawdeh et al., 2009) Table 7 respect1vely.

Table 1. We present the exact and the approximate solutions of the equation in the example 1 in some arbitrary points, the error for $N$ $=10$ is compared with the ones treated in (Babolian and Shahsavaran, 2007)

\begin{tabular}{lllll}
\hline Values of $t$ & Exact solution $\varphi$ & Approx solution $\tilde{\varphi}$ & Error & $\begin{array}{l}\text { Error (Babolian and } \\
\text { Shahsavaran, 2007) }\end{array}$ \\
\hline 0.000000 & $0.00000 \mathrm{e}+00$ & $0.00000 \mathrm{e}+00$ & $0.000 \mathrm{e}+00$ & $0 \mathrm{e}+00$ \\
0.200000 & $2.000000 \mathrm{e}-01$ & $2.000000 \mathrm{e}-01$ & $7.4733 \mathrm{e}-10$ & $4 \mathrm{e}-04$ \\
0.400000 & $4.000000 \mathrm{e}-01$ & $4.000000 \mathrm{e}-01$ & $3.1445 \mathrm{e}-09$ & $6 \mathrm{e}-04$ \\
0.600000 & $6.000000 \mathrm{e}-01$ & $6.000000 \mathrm{e}-01$ & $7.3628 \mathrm{e}-09$ & $9 \mathrm{e}-04$ \\
0.800000 & $8.000000 \mathrm{e}-01$ & $8.000000 \mathrm{e}-01$ & $1.3385 \mathrm{e}-08$ & $1 \mathrm{e}-03$ \\
1.000000 & $1.00000 \mathrm{e}+00$ & $1.00000 \mathrm{e}+00$ & $2.0917 \mathrm{e}-08$ & \\
\hline
\end{tabular}

Table 2. We present the exact and the approximate solutions of the equation in the example 1 in some arbitrary points, the error for $N$ $=10$ is compared with the ones treated in (Maleknejad et al., 2013)

\begin{tabular}{lllll}
\hline & & & & \multicolumn{2}{c}{$\begin{array}{l}\text { Error (Maleknejad } \text { et al., } \\
\text { Values of } t\end{array}$} & Exact solution $\varphi$ & Approx solution $\tilde{\varphi}$ & Error & $2013)$ \\
\hline 0.000000 & $0.00000 \mathrm{e}+00$ & $0.00000 \mathrm{e}+00$ & $0.00 \mathrm{e}+00$ & $0.00 \mathrm{e}+00$ \\
0.200000 & $2.000000 \mathrm{e}-01$ & $2.000000 \mathrm{e}-01$ & $7.47 \mathrm{e}-10$ & $4.22 \mathrm{e}-08$ \\
0.400000 & $4.000000 \mathrm{e}-01$ & $4.000000 \mathrm{e}-01$ & $3.14 \mathrm{e}-09$ & $1.09 \mathrm{e}-08$ \\
0.600000 & $6.000000 \mathrm{e}-01$ & $6.000000 \mathrm{e}-01$ & $7.36 \mathrm{e}-09$ & $2.35 \mathrm{e}-08$ \\
0.800000 & $8.000000 \mathrm{e}-01$ & $8.000000 \mathrm{e}-01$ & $1.33 \mathrm{e}-08$ & $1.42 \mathrm{e}-08$ \\
1.000000 & $1.00000 \mathrm{e}+00$ & $1.00000 \mathrm{e}+00$ & $2.09 \mathrm{e}-08$ & $2.63 \mathrm{e}-08$ \\
\hline
\end{tabular}

Table 3. We present the exact and the approximate solutions of the equation in the example 2 in some arbitrary points, the error for $N$

\begin{tabular}{|c|c|c|c|c|}
\hline Values of $t$ & Exact solution $\varphi$ & Approx solution $\tilde{\varphi}$ & Error & $\begin{array}{l}\text { Error (Abdelwahid, } \\
\text { 2010) }\end{array}$ \\
\hline 0.000000 & $1.000000 \mathrm{e}+00$ & $1.000000 \mathrm{e}+00$ & $0.00 \mathrm{e}+00$ & $0.00 \mathrm{e}+00$ \\
\hline 0.200000 & $1.221403 \mathrm{e}+00$ & $1.221919 \mathrm{e}+00$ & $5.16 \mathrm{e}-04$ & $9.40 \mathrm{e}-04$ \\
\hline 0.400000 & $1.491825 \mathrm{e}+00$ & $1.493531 \mathrm{e}+00$ & $1.70 \mathrm{e}-03$ & $3.06 \mathrm{e}-03$ \\
\hline 0.600000 & $1.822119 \mathrm{e}+00$ & $1.826756 \mathrm{e}+00$ & $4.63 e-03$ & $8.16 \mathrm{e}-03$ \\
\hline 0.800000 & $2.225541 \mathrm{e}+00$ & $2.238233 \mathrm{e}+00$ & $1.26 \mathrm{e}-02$ & $2.16 \mathrm{e}-02$ \\
\hline 1.000000 & $2.718282 \mathrm{e}+00$ & $2.756934 \mathrm{e}+00$ & $3.86 \mathrm{e}-02$ & $6.27 \mathrm{e}-02$ \\
\hline
\end{tabular}
$=10$ is compared with the ones treated in (Abdelwahid, 2010) 
Table 4. We present the exact and the approximate solutions of the equation in the example 3 in some arbitrary points, the error for $N$ $=10$ is compared with the ones treated in (Shahsavaran, 2011)

\begin{tabular}{lllll}
\hline Values of $t$ & Exact solution $\varphi$ & Approx solution $\tilde{\varphi}$ & \multicolumn{2}{c}{$\begin{array}{l}\text { Error (Shahsavaran, } \\
\text { Error }\end{array}$} \\
\hline 0.000000 & $0.00000 \mathrm{e}+00$ & $0.00000 \mathrm{e}+00$ & $0.00 \mathrm{e}+00$ & $0.0 \mathrm{e}+00$ \\
0.200000 & $1.986693 \mathrm{e}-01$ & $1.986672 \mathrm{e}-01$ & $2.11 \mathrm{e}-06$ & $5.4 \mathrm{e}-03$ \\
0.400000 & $3.894183 \mathrm{e}-01$ & $3.894008 \mathrm{e}-01$ & $1.75 \mathrm{e}-05$ & $5.8 \mathrm{e}-03$ \\
0.600000 & $5.646425 \mathrm{e}-01$ & $5.645828 \mathrm{e}-01$ & $5.97 \mathrm{e}-05$ & $7.0 \mathrm{e}-03$ \\
0.800000 & $7.173561 \mathrm{e}-01$ & $7.172144 \mathrm{e}-01$ & $1.41 \mathrm{e}-04$ & $4.1 \mathrm{e}-03$ \\
1.000000 & $8.414710 \mathrm{e}-01$ & $8.411949 \mathrm{e}-01$ & $2.76 \mathrm{e}-04$ & \\
\hline
\end{tabular}

Table 5. We present the exact and the approximate solutions of the equation in the example 4 in some arbitrary points, the error for $N$ $=10$ is compared with the ones treated in (Lepik and Tamme, 2007)

\begin{tabular}{lllll}
\hline Values of $\mathrm{t}$ & Exact solution $\varphi$ & Appro solution $\tilde{\varphi}$ & Error & $\begin{array}{l}\text { Error (Lepik and } \\
\text { Tamme, 2007) }\end{array}$ \\
\hline 0.000000 & $0.00000 \mathrm{e}+00$ & $0.00000 \mathrm{e}+00$ & $0.00 \mathrm{e}+00$ & $2.7 \mathrm{e}-04$ \\
0.200000 & $4.472136 \mathrm{e}-01$ & $4.472137 \mathrm{e}-01$ & $1.10 \mathrm{e}-07$ & $2.7 \mathrm{e}-04$ \\
0.400000 & $6.324555 \mathrm{e}-01$ & $6.324560 \mathrm{e}-01$ & $4.40 \mathrm{e}-07$ & $2.7 \mathrm{e}-04$ \\
0.600000 & $7.745967 \mathrm{e}-01$ & $7.745977 \mathrm{e}-01$ & $9.90 \mathrm{e}-07$ & $2.7 \mathrm{e}-04$ \\
0.800000 & $8.944272 \mathrm{e}-01$ & $8.944290 \mathrm{e}-01$ & $1.76 \mathrm{e}-06$ & $2.7 \mathrm{e}-04$ \\
1.000000 & $1.00000 \mathrm{e}+00$ & $1.00000 \mathrm{e}+00$ & $2.75 \mathrm{e}-06$ & $2.7 \mathrm{e}-04$ \\
\hline
\end{tabular}

Table 6. We present the exact and the approximate solutions of the equation in the example 5 in some arbitrary points, the error for $N$ $=10$ is compared with the ones treated in (Saberi-Nadja and Heidari, 2010)

\begin{tabular}{lllll}
\hline Values of $\mathrm{t}$ & Exact solution $\varphi$ & Approx solution $\tilde{\varphi}$ & Error & $\begin{array}{c}\text { Error (Saberi-Nadja and } \\
\text { Heidari, 2010) }\end{array}$ \\
\hline 0.000000 & $7.542669 \mathrm{e}-02$ & $7.542663 \mathrm{e}-02$ & $5.44 \mathrm{e}-08$ & $4.98 \mathrm{e}-02$ \\
0.200000 & $6.488067 \mathrm{e}-01$ & $6.488067 \mathrm{e}-01$ & $4.40 \mathrm{e}-08$ & $4.03 \mathrm{e}-02$ \\
0.400000 & $9.743646 \mathrm{e}-01$ & $9.743646 \mathrm{e}-01$ & $1.68 \mathrm{e}-08$ & $1.53 \mathrm{e}-02$ \\
0.600000 & $9.277484 \mathrm{e}-01$ & $9.277484 \mathrm{e}-01$ & $1.68 \mathrm{e}-08$ & $1.53 \mathrm{e}-02$ \\
0.800000 & $5.267638 \mathrm{e}-01$ & $5.267638 \mathrm{e}-01$ & $4.40 \mathrm{e}-08$ & $4.03 \mathrm{e}-02$ \\
1.000000 & $-7.542669 \mathrm{e}-02$ & $-7.542663 \mathrm{e}-02$ & $5.44 \mathrm{e}-08$ & $1.53 \mathrm{e}-02$ \\
\hline
\end{tabular}

Table 7. We present the exact and the approximate solutions of the equation in the example 5 in some arbitrary points, the error for $N$ $=20$ is compared with the ones treated in (Awawdeh et al., 2009)

\begin{tabular}{lllll}
\hline Values of $\mathrm{t}$ & Exact solution $\varphi$ & Approx solution $\tilde{\varphi}$ & Error & $\begin{array}{l}\text { Error (Awawdeh } \text { et al., } \\
\text { 2009) }\end{array}$ \\
\hline 0.000000 & $7.542669 \mathrm{e}-002$ & $7.542669 \mathrm{e}-002$ & $3.19 \mathrm{e}-016$ & $5.53 \mathrm{e}-015$ \\
0.200000 & $6.488067 \mathrm{e}-001$ & $6.488067 \mathrm{e}-001$ & $2.22 \mathrm{e}-016$ & $4.55 \mathrm{e}-015$ \\
0.400000 & $9.743646 \mathrm{e}-001$ & $9.743646 \mathrm{e}-001$ & $1.11 \mathrm{e}-016$ & $1.77 \mathrm{e}-015$ \\
0.600000 & $9.277484 \mathrm{e}-001$ & $9.277484 \mathrm{e}-001$ & $1.11 \mathrm{e}-016$ & $1.77 \mathrm{e}-015$ \\
0.800000 & $5.267638 \mathrm{e}-001$ & $5.267638 \mathrm{e}-001$ & $2.22 \mathrm{e}-016$ & $4.55 \mathrm{e}-015$ \\
1.000000 & $-7.542669 \mathrm{e}-002$ & $-7.542669 \mathrm{e}-002$ & $3.19 \mathrm{e}-016$ & $5.53 \mathrm{e}-015$ \\
\hline
\end{tabular}

\section{Acknowledgment}

The authors are grateful to the anonymous referees who have contributed to improve the quality of this paper.

\section{Author's Contributions}

The authors contributed equally to the writing of this paper and they read and approved the final manuscript.

\section{Ethics}

Authors address any ethical issues that may arise after the publication of this manuscript.

\section{References}

Abdelwahid, F., 2010. Adomian decomposition method applied to nonlinear integral equations. Alexandria J. Math., 1: 11-18. 
Awawdeh, F., A. Adawi and S. Al-Shara, 2009. A numerical method for solving nonlinear integral equations. Int. Math. Forum, 4: 805-817.

Babolian, E. and A. Shahsavaran, 2007. Numerical solution of nonlinear Fredholm and Volterra integral equations of the second kind using Haar wavelets and collocation method. J. Sci. Tarbiat Moallem Univ., 7: 213.222.

Golberg, M.A., 1990. Numerical Solution of Integral Equations. 1st Edn., Springer, New York, ISBN-10: 0306432625, pp: 418.

Lepik, U. and E. Tamme, 2007. Solution of nonlinear Fredholm integral equations via the Haar wavelet method. Proc. Estonian Acad. Sci. Phys. Math., 56: 17-27.

Maleknejad, K., R. Mollapourasl and M. Shahabi, 2013. On the solution of a nonlinear integral equation on the basis of a fixed point technique and cubic Bspline scaling functions. J. Comput. Applied Math., 239: 346-358. DOI: 10.1016/j.cam.2012.09.002

Nadir, M. and B. Gagui, 2014. A numerical approximation for solutions of Hammerstein integral equations in $\mathrm{L}^{\mathrm{p}}$ spaces. Sao Paulo J. Math. Sci., 8: 23-33.
Nadir, M. and A. Rahmoune, 2007. Modified method for solving linear volterra integral equations of the second kind using Simpsons rule. Int. J. Math. Manuscripts, 1: 133-140.

Polyanin, A.D. and A.V. Manzhirov, 2008. Handbook of Integral Equations. 2nd Edn., CRC Press, ISBN-10: 0203881052, pp: 1144.

Saberi-Nadja, J. and M. Heidari, 2010. Solving nonlinear integral equations in the Urysohn form by NewtonKantorovich-quadrature method. Comput. Math. Applic., 60: 2058-2065.

DOI: 10.1016/j.camwa.2010.07.046

Shahsavaran, A., 2011. Computational method to solve nonlinear integral equations using block pulse functions by collocation method. Applied Math. Sci., 5: 3211-3220.

Wouk, A., 1979. A course of Applied Functional Analysis. 1st Edn., Wiley, New York, ISBN-10: 0471962384, pp: 443. 